\title{
LOCALLY SYMMETRIC AFFINE HYPERSURFACES
}

\author{
P. VERHEYEN AND L. VERSTRAELEN
}

\begin{abstract}
The nondegenerate quadratic hypersurfaces and the improper affine hyperspheres are the only nondegenerate hypersurfaces of dimension greater than two of an affine space which are affine locally symmetric with respect to their induced connection.
\end{abstract}

1. Introduction. Let $\mathbf{A}^{n+1}$ be an $(n+1)$-dimensional affine space considered as a homogeneous space under action of the unimodular affine group $\operatorname{ASL}(n+1, \mathbf{R})(n \geq 2)$. $\quad \mathbf{A}^{n+1}$ has a natural equiaffine structure $(\tilde{\nabla}, \tilde{\theta})$, i.e. a torsion-free affine connection $\tilde{\nabla}$ and a volume element $\tilde{\theta}$ which is parallel w.r.t. this connection. We now briefly indicate how one gets an induced equiaffine structure $(\nabla, \theta)$ on a nondegenerate hypersurface of $\mathbf{A}^{n+1}$ (see $\left.[\mathbf{2}, \mathbf{4}, \mathbf{5}, \mathbf{7}]\right)$.

Let $M^{n}$ be a hypersurface of $\mathbf{A}^{n+1}$. Denote the set of all vector fields which are transversal to $M$ by $\mathfrak{X}_{\mathrm{tr}} M$. For each vector field $\xi$ in $\mathfrak{X}_{\mathrm{tr}} M$ we have an induced volume element $\theta^{\xi}$ on $M$, defined by

$$
\theta^{\xi}\left(X_{1}, \ldots, X_{n}\right):=\tilde{\theta}\left(X_{1}, \ldots, X_{n}, \xi\right)
$$

for vector fields $X_{1}, \ldots, X_{n}$ tangent to $M$. Moreover, by decomposing $\tilde{\nabla}_{X} Y$ for vector fields $X$ and $Y$ tangent to $M$ in a tangential component $\nabla_{X}^{\xi} Y$ and in a component $h^{\xi}(X, Y) \xi$ in the direction of $\xi$, we get a torsion-free affine connection $\nabla^{\xi}$ on $M$ and a bilinear map $h^{\xi}: T_{x} M \times T_{x} M \rightarrow \mathbf{R}$ at each point $x$ of $M$. In the same way we can decompose $\tilde{\nabla}_{X} \xi$ for $X$ in $\mathfrak{X} M$ in a tangential component $\left(-S^{\xi} X\right)$ and in a component in the direction of $\xi\left(\tau^{\xi}(X) \xi\right)$. This defines a linear map $S^{\xi}: T_{x} M \rightarrow T_{x} M$ at each point $x$ of $M$ and a 1 -form $\tau^{\xi}$ on $M$. The pair $\left(\nabla^{\xi}, \theta^{\xi}\right)$ is not necessarily an equiaffine structure on $M$ because $\nabla^{\xi} \theta^{\xi}=\tau^{\xi} \otimes \theta^{\xi}$. In the case where $M$ is a nondegenerate hypersurface (i.e. $h^{\xi}$ is nondegenerate for one transversal vector field $\xi$, which implies that this is true for all $\xi$ in $\mathfrak{X}_{\mathrm{tr}} M$ ), it is possible to find transversal vector fields $\xi$ for which $\tau^{\xi}=0$. Let $\Gamma$ denote the set of all $\xi$ in $\mathfrak{X}_{\operatorname{tr}} M$ satisfying $\tau^{\xi}=0$ and let $\mathfrak{V}$ be the set of all volume elements on $M$. Then the map $\Gamma \rightarrow \mathfrak{V}: \xi \mapsto \theta^{\xi}$ is injective. We now construct an affinely invariant element $\hat{\nu}$ of $\mathfrak{V}$ which lies in the range of this map $\Gamma \rightarrow \mathfrak{V}$. The unique transversal vector field in $\Gamma$ mapped to $\hat{\nu}$ is called the affine normal and this affine normal gives rise to an induced equiaffine structure on $M$.

To construct $\hat{\nu}$, take an arbitrary $\xi$ in $\mathfrak{X}_{\mathrm{tr}} M$. Then $h^{\xi}$ is nondegenerate and we can associate with it an affinely invariant pseudo-Riemannian metric $\hat{h}$ as follows. Choose vector fields $e_{1}, \ldots, e_{n}$ tangent to $M$ and satisfying $\tilde{\theta}\left(e_{1}, \ldots, e_{n}, \xi\right)=1$.

Received by the editors September 15, 1983.

1980 Mathematics Subject Classification. Primary 53C40, 53A15.

Key words and phrases. Nondegenerate affine hypersurfaces, quadratic hypersurfaces, affine hyperspheres, affine locally symmetric spaces.

(c) 1985 American Mathematical Society $0002-9939 / 85 \$ 1.00+\$ .25$ per page 
Then

$$
\hat{h}=\left|\operatorname{det}\left(h^{\xi}\left(e_{i}, e_{j}\right)\right)\right|^{1 / n+2} h^{\xi}
$$

is independent of the choice of $\xi$ in $\mathfrak{X}_{\mathrm{tr}} M$ and of the choice of $e_{1}, \ldots, e_{n}$. Hence $\hat{h}$ gives a volume element $\hat{\nu}$ and this $\hat{\nu}$ lies in the range of the map $\Gamma \rightarrow \mathfrak{V}: \xi \mapsto \theta^{\xi}$.

From now on we suppose $M$ to be a nondegenerate hypersurface of $\mathbf{A}^{n+1}$ with affine normal $\xi$. We delete $\xi$ from all notations (e.g. $h=h^{\xi}=\hat{h}, \theta=\theta^{\xi}=\hat{\nu}$ ), thus having

$$
\tilde{\nabla}_{X} Y=\nabla_{X} Y+h(X, Y) \xi
$$

and

$$
\tilde{\nabla}_{X} \xi=-S X
$$

for all vector fields $X$ and $Y$ tangent to $M . \alpha=\frac{1}{n} \operatorname{Tr} S$ is called the affine mean curvature of $M$.

We now give the fundamental equations of a nondegenerate hypersurface $M$ in $\mathbf{A}^{n+1}[\mathbf{7}]$. Let $X, Y$ and $Z$ be vector fields tangent to $M$. Then the equation of Gauss is given by

$$
R(X, Y) Z=h(Y, Z) S X-h(X, Z) S Y
$$

where $R$ is the curvature tensor of $(M, \nabla)$, the equations of Codazzi state that

$$
\left(\nabla_{X} h\right)(Y, Z)=\left(\nabla_{Y} h\right)(X, Z)
$$

and

$$
\left(\nabla_{X} S\right) Y=\left(\nabla_{Y} S\right) X
$$

and the equation of Ricci is given by

$$
h(X, S Y)=h(S X, Y)
$$

The form $\Psi$ defined by $\Psi=\nabla h$ is called the cubic form of $M$.

The pseudo-Riemannian metric $h$ gives rise to the uniquely determined pseudoRiemannian connection $\hat{\nabla}$ which is torsion-free and satisfies $\hat{\nabla} h=0$. Using this connection the affine normal $\xi$ is given by $\xi=\frac{1}{n} \hat{\Delta} x$, where $\hat{\Delta}$ is the Laplacian of $(M, \hat{\nabla})$ and $x$ is the position vector field in $\mathbf{A}^{n+1}$. We mention the following result of L. Berwald concerning the case where $\nabla=\hat{\nabla}$.

Proposition $1[\mathbf{1}, 5]$. Let $M^{n}$ be a nondegenerate hypersurface in $\mathbf{A}^{n+1}$ $(n \geq 2)$. Then $\Psi=0$ if and only if $M$ is a nondegenerate quadratic hypersurface.

The nondegenerate quadratic hypersurfaces are examples of the so-called affine hyperspheres which are defined by the condition that the shape-operator $S$ is a multiple of the identity. We remark that in this case $S=\alpha I$, where $\alpha$ is constant (see (1.6)). Alternatively, the affine hyperspheres can be defined by the condition that their affine normals are parallel $(S=0)$ or all pass through a fixed point, which is called the center of $M\left(S=\alpha I, \alpha \in \mathbf{R}_{0}\right)$. An affine hypersphere of the first type is called an improper affine hypersphere, whereas one of the second type is said to be a proper affine hypersphere. For convex proper affine hyperspheres one can make a distinction between those of elliptic type and those of hyperbolic type depending upon the fact that the center lies on the convex side of $M$ or not. E.g. 
$\left\{x \in \mathbf{A}^{n+1} \mid x^{1} \cdots x^{n+1}=1\right.$ with $\left.\forall i \in\{1, \ldots, n+1\}: x^{i} \in \mathbf{R}_{0}^{+}\right\}$is a proper affine hypersphere of the hyperbolic type. The convex affine hyperspheres in $\mathbf{A}^{n+1}$ which are complete (w.r.t. the Riemannian metric $h$ ) are completely classified for $n \geq 2$ (see $[3,6])$.

An example of a nonconvex affine hypersphere is given by the imbedding of $\mathrm{SL}(n, \mathbf{R})$ in $\mathrm{GL}^{+}(n, \mathbf{R}) \cong \mathbf{A}^{n^{2}}$. In particular for $n=2$ this gives a quadratic hypersurface in $\mathbf{A}^{4}$ such that $\nabla R=0[\mathbf{7}]$. In [7] K. Nomizu asks for other examples of nondegenerate hypersurfaces which are affine locally symmetric w.r.t. the induced connection $\nabla$. We will prove that there are, in fact, no nontrivial examples of this kind when $n>2$.

THEOREM. Let $M^{n}$ be a nondegenerate hypersurface of $\mathbf{A}^{n+1}$ with $n>2$. Then $M$ is affine locally symmetric w.r.t. the induced connection $\nabla$ if and only if $M$ is an improper affine hypersphere or a nondegenerate quadratic hypersurface.

2. Proof of the Theorem. Let $M$ ba a nondegenerate hypersurface in $\mathbf{A}^{n+1}$. If $\nabla R=0$, then $M$ also satisfies the condition $R \cdot R=0$, where $R$ acts on $R$ as a derivation. Let $X, Y, Z, V$ and $W$ be vectors tangent to $M$. Then (1.4) implies

$$
\begin{aligned}
(R(X, Y) \cdot R) & (Z, V) W=[h(V, W) h(Y, S Z)-h(Z, W) h(Y, S V)] S X \\
& +[h(Z, W) h(X, S V)-h(V, W) h(X, S Z)] S Y \\
& +[h(Y, V) h(Z, W)-h(Y, Z) h(V, W)] S^{2} X \\
& +[h(X, Z) h(V, W)-h(X, V) h(Z, W)] S^{2} Y \\
& +[h(X, V) h(S Y, W)-h(Y, V) h(S X, W) \\
& +h(X, W) h(S Y, V)-h(Y, W) h(S X, V)] S Z \\
& +[h(Y, Z) h(S X, W)-h(X, Z) h(S Y, W) \\
& +h(Y, W) h(S X, Z)-h(X, W) h(S Y, Z)] S V .
\end{aligned}
$$

First, we consider the case in which $S$ and $h$ are simultaneously diagonalizable. This is certainly true for a convex hypersurface, but (1.7) does not imply the same result for an arbitrary hypersurface (see e.g. the endomorphism $S: \mathbf{R}^{n} \rightarrow \mathbf{R}^{n}:\left(x^{1}, \ldots, x^{s-1}, x^{s}, x^{s+1}, x^{s+2}, \ldots, x^{n}\right) \mapsto\left(x^{1}, \ldots, x^{s-1}, x^{s}-x^{s+1}, x^{s}+\right.$ $\left.x^{s+1}, x^{s+2}, \ldots, x^{n}\right)$ and the pseudo-Riemannian metric $h=\sum_{i=1}^{s} d x^{i} \otimes d x^{i}-$ $\left.\sum_{j=s+1}^{n} d x^{j} \otimes d x^{j}\right)$. If $S$ and $h$ can be put simultaneously in diagonal form, then we have the following

LEMMA 1. Let $M$ be a nondegenerate hypersurface in $\mathbf{A}^{n+1}$ and $x \in M$. If there exists a basis of $T_{x} M$ consisting of eigenvectors of $S$, then there exists a basis $\left(e_{1}, \ldots, e_{n}\right)$ of $T_{x} M$ such that $S e_{i}=\rho_{i} e_{i}$ with $h\left(e_{i}, e_{j}\right)=\varepsilon_{i} \delta_{i j}$, where $\varepsilon_{i} \in\{-1,1\}$ and $i, j \in\{1, \ldots, n\}$.

PROOF. Let $\left(u_{1}, \ldots, u_{n}\right)$ be a basis of $T_{x} M$ satisfying $S u_{i}=\rho_{i} u_{i}$. Then $\left(\rho_{i}-\rho_{j}\right) h\left(u_{i}, u_{j}\right)=0$. Suppose $h\left(u_{i}, u_{i}\right)=0$ for $1 \leq i \leq l$ and $h\left(u_{j}, u_{j}\right) \neq 0$ for $l+1 \leq j \leq n$, such that $h\left(u_{j}, u_{j}\right)=\varepsilon_{j}$ for $l+1 \leq j \leq n$ and $l \in \mathbf{N}_{0}$. We show that one can reduce the number $l$.

If there exist $i$ and $j$ such that $1 \leq i \leq l<j \leq n$ and $\rho_{i}=\rho_{j}$, then there exist vectors $\bar{u}_{i}$ and $\bar{u}_{j}$ in the plane spanned by $u_{i}$ and $u_{j}$ satisfying $h\left(\bar{u}_{i}, \bar{u}_{i}\right) \neq 0 \neq$ $h\left(\bar{u}_{j}, \bar{u}_{j}\right), S \bar{u}_{i}=\rho_{i} \bar{u}_{i}$ and $S \bar{u}_{j}=\rho_{j} \bar{u}_{j}$. Hence we may suppose that $\rho_{i} \neq \rho_{j}$ and 
$h\left(u_{i}, u_{j}\right)=0$ for all $i$ and $j$ satisfying $1 \leq i \leq l<j \leq n$. Moreover $l \geq 2$ and at least one of the $h\left(u_{1}, u_{i}\right), 1<i \leq l$, is different from zero (otherwise $u_{1}=0$ ). Suppose $h\left(u_{1}, u_{2}\right) \neq 0$. Then $\rho_{1}=\rho_{2}$ and there exist vectors $\bar{u}_{1}$ and $\bar{u}_{2}$ in the plane spanned by $u_{1}$ and $u_{2}$ such that $h\left(\bar{u}_{1}, \bar{u}_{1}\right) \neq 0 \neq h\left(\bar{u}_{2}, \bar{u}_{2}\right), S \bar{u}_{1}=\rho_{1} \bar{u}_{1}$ and $S \bar{u}_{2}=\rho_{2} \bar{u}_{2}$. This proves Lemma 1 .

LEMMA 2. Let $M^{n}$ be a nondegenerate hypersurface in $\mathbf{A}^{n+1}$ with $n>2$. If $S$ is diagonalizable, then $R \cdot R=0$ if and only if $M$ is an affine hypersphere.

PROOF. It follows from (2.1) that each affine hypersphere satisfies the condition $R \cdot R=0$. Next, for a nondegenerate hypersurface, suppose that $S$ is diagonalizable and $R \cdot R=0$. Let $x$ be a point of $M$ and let $\left(e_{1}, \ldots, e_{n}\right)$ be a basis of $T_{x} M$ satisfying $S e_{i}=\rho_{i} e_{i}$ and $h\left(e_{i}, e_{j}\right)=\varepsilon_{i} \delta_{i j}$ with $\varepsilon_{i} \in\{-1,1\}$. We agree that indices $i, j, k, l$ will always be in the range $1, \ldots, n$.

For three mutually distinct indices $i, j, k$ the condition $\left(R\left(e_{j}, e_{i}\right) \cdot R\right)\left(e_{k}, e_{i}\right) e_{k}=0$ implies that $0=\varepsilon_{i} \varepsilon_{k} \rho_{j}\left(\rho_{i}-\rho_{j}\right) e_{j}$. Consequently $\rho_{j}\left(\rho_{i}-\rho_{j}\right)=0$ for $i \neq j$. If there exists a $\rho_{l}$ different from zero, then all other $\rho_{i}$ are equal to this $\rho_{l}$ and $S=\alpha I$. In the other case $S=0$.

We now consider the general case in which $S$ is not necessarily diagonalizable.

Proposition 2. Let $M^{n}$ be a nondegenerate hypersurface in $\mathbf{A}^{n+1}$ with $n>2$. Then $R \cdot R=0$ if and only if $M$ is an affine hypersphere.

Proof. We will always work in $T_{x} M$, where $x$ is a point of $M$. Suppose $R \cdot R=0$. Then for all vectors $X, Y$ and $Z$ in $T_{x} M$ satisfying $h(X, Y)=0=$ $h(X, Z)=h(Y, Z)$ we have

$$
[h(X, X) h(S Y, Y)-h(Y, Y) h(S X, X)] S Z+h(Y, Y) h(S X, Z) S X=0 .
$$

We consider two cases: $\operatorname{dim}(\operatorname{im} S) \geq 2$ and $\operatorname{dim}(\operatorname{im} S)=1($ if $\operatorname{dim}(\operatorname{im} S)=0$ then $S=0)$.

Case 1 . When $\operatorname{dim}(\operatorname{im} S) \geq 2$, we may suppose that $\left(e_{1}, \ldots, e_{n}\right)$ is a basis of $T_{x} M$ such that $h\left(e_{i}, e_{j}\right)=\varepsilon_{i} \delta_{i j}$ and $S e_{1}$ and $S e_{2}$ are linearly independent $\left(\varepsilon_{i} \in\{-1,1\}\right.$; $i, j, k, l \in\{1, \ldots, n\})$.

Taking $X=e_{1}, Z=e_{2}$ and $Y=e_{i}$ for $2<i \leq n$ in (2.2), we have $\varepsilon_{1} \varepsilon_{i}\left(S_{i}^{i}-S_{1}^{1}\right)=$ 0 and $\varepsilon_{i} \varepsilon_{2} S_{1}^{2}=0$, where $S_{k}^{l}$ is defined by $S e_{k}=\sum_{k=1}^{n} S_{k}^{l} e_{l}$. Consequently, we have $S_{1}^{2}=0$ and $S_{1}^{1}=S_{i}^{i}$ for $2<i \leq n$.

Taking $X=e_{2}, Z=e_{1}$ and $Y=e_{i}$ for $2<i \leq n$, we also have $S_{2}^{2}=S_{i}^{i}$, i.e. $S_{j}^{j}=$ $\rho$ for all $j$ in $\{1, \ldots, n\}$. This implies that $h\left(e_{i}, e_{i}\right) h\left(S e_{j}, e_{j}\right)=h\left(e_{j}, e_{j}\right) h\left(S e_{i}, e_{i}\right)$ for all $i$ and $j$.

For mutually distinct $i, j$ and $k$, it follows from (2.2) with $X=e_{i}, Y=e_{j}$ and $Z=e_{k}$ that $\varepsilon_{j} \varepsilon_{k} S_{i}^{k}\left(S e_{i}\right)=0$. Consequently $S_{i}^{j}=0$ for all $i$ and $j$ with $i \neq j$. Lemma 2 then proves Proposition 2.

Case 2. Suppose im $S$ is generated by a vector $u \neq 0$. If $h(u, u) \neq 0$, then we may suppose that $\left(e_{1}, \ldots, e_{n}\right)$ is a basis of $T_{x} M$ such that $h\left(e_{i}, e_{j}\right)=\varepsilon_{i} \delta_{i j}$ with $\varepsilon_{i} \in\{-1,1\}$ and $u=e_{1}$, i.e. $S e_{i}=\rho_{i} e_{1}$. In this case (2.2) implies for $X=e_{1}$, $Y=e_{i}$ and $Z=e_{j}$, where $1<i, 1<j$ and $i \neq j$, that $\varepsilon_{1} \varepsilon_{i} \rho_{1} \rho_{j} e_{1}=0$. Hence $\rho_{1} \rho_{i}=0$ for all $i$ greater than 1. If $\rho_{1} \neq 0$, then all other $\rho_{i}$ are equal to zero and $S$ is diagonalizable. Lemma 2 then implies that $S=0$ which gives a contradiction. 
So $\rho_{1}=0$. Taking $X=e_{i}, Y=e_{j}$ and $Z=e_{1}(1<i, 1<j$ and $i \neq j)$ in (2.2), we get $\rho_{i}^{2}=0$, i.e. $S=0$. This gives again a contradiction.

Let $\left(e_{1}, \ldots, e_{n}\right)$ be a basis of $T_{x} M$ such that $h\left(e_{i}, e_{j}\right)=\varepsilon_{i} \delta_{i j}\left(\varepsilon_{i} \in\{-1,1\}\right)$ and suppose $S e_{i}=\rho_{i} u$ with $u \neq 0$ and $h(u, u)=0$. Formula (1.7) implies that

$$
\rho_{i} h\left(u, e_{j}\right)=\rho_{j} h\left(u, e_{i}\right)
$$

for all $i$ and $j$. Let $i, j$ and $k$ be mutually distinct and put $X=e_{i}, Y=e_{j}$ and $Z=e_{k}$ in $(2.2)$. Then

$$
\begin{aligned}
0 & =\left[\varepsilon_{i} \rho_{j} h\left(u, e_{j}\right)-\varepsilon_{j} \rho_{i} h\left(u, e_{i}\right)\right] \rho_{k}+\varepsilon_{j} \rho_{i} h\left(u, e_{k}\right) \rho_{i} \\
& =\varepsilon_{i} \rho_{j} \rho_{k} h\left(u, e_{j}\right)+\varepsilon_{j} \rho_{i}\left[\rho_{i} h\left(u, e_{k}\right)-\rho_{k} h\left(u, e_{i}\right)\right] \\
& =\varepsilon_{i} \rho_{j}^{2} h\left(u, e_{k}\right)
\end{aligned}
$$

because of (2.3). Consequently, $\rho_{i}^{2} h\left(u, e_{j}\right)=0$ for all $i$ and $j$ which are distinct. Now there exist at least two $h\left(u, e_{j}\right)$ which are different from zero (otherwise $u=$ $\sum_{j=1}^{n} \varepsilon_{j} h\left(u, e_{j}\right) e_{j}$ would not satisfy $\left.h(u, u)=0\right)$. Hence all $\rho_{i}$ are equal to zero and $S=0$. This gives again a contradiction and thus Case 2 cannot occur.

PROOF OF THEOREM. Let $M$ be a nondegenerate hypersurface in $\mathbf{A}^{n+1}$ with $n>2$. If $\nabla R=0$, then also $R \cdot R=0$ and $M$ is an affine hypersphere, i.e. $S=\alpha I$, where $\alpha$ is constant. From (1.4) we have

$$
\left(\nabla_{V} R\right)(X, Y) Z=\alpha\left[\left(\nabla_{V} h\right)(Y, Z) X-\left(\nabla_{V} h\right)(X, Z) Y\right]
$$

such that $\nabla R=0$ if and only if $\alpha=0$ or $\nabla h=0$. Proposition 1 then proves the Theorem.

REMARK. Each surface $M^{2}$ in $\mathbf{A}^{3}$ satisfies the condition $R \cdot R=0$. Using the fundamental equations for a surface in $\mathbf{A}^{3}$, one can prove that a nondegenerate surface $M$ in $\mathbf{A}^{3}$ for which $S$ is diagonalizable and which has constant affine mean curvature is an improper affine hypersphere or a nondegenerate quadratic surface if $\nabla R=0$. In particular, a nondegenerate complete convex surface in $\mathbf{A}^{3}$ with constant affine mean curvature is affine locally symmetric w.r.t. the induced connection if and only if it is a nondegenerate quadratic surface.

\section{REFERENCES}

1. L. Berwald, Die Grundgleichungen der Hyperflächen im Euklidischen $R_{n+1}$ gegenüber den inhaltstreuen Affinitäten, Monatsh. Math. Phys. 32 (1922), 89-106.

2. W. Blaschke, Vorlesungen über Differentialgeometrie, Chelsea, New York, 1967.

3. E. Calabi, Géométrie différentielle affine des hypersurfaces, Séminaire Bourbaki 1980/81, n 573 , Lecture Notes in Math., vol. 901, Springer-Verlag, Berlin, Heidelberg and New York, 1981 , pp. 189-204.

4. S.-S. Chern, Affine minimal hypersurfaces, Minimal Submanifolds and Geodesics, Tokyo, 1978 , pp. 17-30.

5. H. Flanders, Local theory of affine hypersurfaces, J. Analyse Math. 15 (1965), 353-387.

6. S. Gigena, On a conjecture by E. Calabi, Geom. Dedicata 11 (1981), 378-396.

7. K. Nomizu, What is affine differential geometry? Proc. Differential Geom. (Münster, 1982), 42-43.

Katholieke Universiteit Leuven, FaCUlteit Wetenschappen, Departement Wiskunde, CelestijnenlaAn 200 B, B-3030 Leuven, Belgium 